\title{
Universiteit
}

Leiden

The Netherlands

\section{Dynamic ordering of driven vortex matter in the peak effect regime of amorphous MoGe films and $2 \mathrm{H}-\mathrm{NbSe} 2$ crystals}

Kokubo, N.; Asada, T.; Kadowaki, K.; Takita, K.; Sorop, T.G.; Kes, P.H.

\section{Citation}

Kokubo, N., Asada, T., Kadowaki, K., Takita, K., Sorop, T. G., \& Kes, P. H. (2007). Dynamic ordering of driven vortex matter in the peak effect regime of amorphous MoGe films and 2HNbSe2 crystals. Physical Review B, 75(18), 184512. doi:10.1103/PhysRevB.75.184512

Version: $\quad$ Not Applicable (or Unknown)

License: $\quad$ Leiden University Non-exclusive license

Downloaded from: https://hdl.handle.net/1887/77072

Note: To cite this publication please use the final published version (if applicable). 


\title{
Dynamic ordering of driven vortex matter in the peak effect regime of amorphous MoGe films and $2 \mathrm{H}-\mathrm{NbSe}_{2}$ crystals
}

\author{
N. Kokubo, ${ }^{1, *}$ T. Asada, ${ }^{1}$ K. Kadowaki, ${ }^{1}$ K. Takita, ${ }^{1}$ T. G. Sorop, ${ }^{2}$ and P. H. Kes ${ }^{2}$ \\ ${ }^{1}$ Institute of Materials Science, University of Tsukuba, 1-1-1, Tennoudai, Tsukuba, Ibaraki 305-8573, Japan \\ ${ }^{2}$ Kamerlingh Onnes Laboratory, Leiden University, P.O. Box 9504, 2300 RA Leiden, The Netherlands
}

(Received 2 December 2006; published 15 May 2007)

\begin{abstract}
Dynamic ordering of driven vortex matter has been investigated in the peak effect regime of both amorphous MoGe films and $2 \mathrm{H}-\mathrm{NbSe}_{2}$ crystals by mode locking (ML) and dc transport measurements. ML features allow us to trace how the shear rigidity of driven vortices evolves with the average velocity. Determining the onset of ML resonance in different magnetic fields and/or temperatures, we find that the dynamic ordering frequency (velocity) exhibits a striking divergence in the higher part of the peak effect regime. Interestingly, this phenomenon is accompanied by a pronounced peak of dynamic critical current. Mapping out field-temperature phase diagrams, we find that divergent points follow well the thermodynamic melting curve of the ideal vortex lattice over wide field and/or temperature ranges. These findings provide a link between the dynamic and static melting phenomena which can be distinguished from the disorder induced peak effect.
\end{abstract}

DOI: $10.1103 /$ PhysRevB.75.184512

PACS number(s): 74.25.Qt, 74.25.Sv, 74.25.Fy

\section{INTRODUCTION}

Vortex matter in type-II superconductors has been recognized as an ideal system for studying collective transports of periodic manifolds driven over the pinning environments. ${ }^{1,2}$ In particular, much recent attention has focused on the issue of dynamic ordering, separating elasticity dominated, coherent lattice flow at large velocity from plastically deformed, incoherent defective flow at small velocity. Theoretical considerations for this phenomenon were first proposed by Koshelev and Vinokur by introducing a concept of shaking temperature, which characterizes fluctuating motion of vortices due to the interaction with disorder quenched in a host material. ${ }^{3}$ Assuming the shaking temperature to decay as $k_{B} T_{s h}=\Gamma / v$ on increasing the average velocity $v$, they proposed that the ordering (crystallization) velocity diverges as $v_{c} \propto \Gamma / k_{B}\left(T_{M}-T\right)$ with a disorder parameter $\Gamma$ on approaching the thermal melting temperature $T_{M}$ from below. Later, more sophisticated theoretical ${ }^{4-7}$ and numerical studies ${ }^{8-13}$ have suggested various coherent flow states with different positional and orientational orders such as moving glass state and smectic flow state, and some of them have proposed multiple transitions in ordering process.

While experimental evidences for the ordering were reported in neutron-diffraction and Bitter decoration studies, ${ }^{14-17}$ investigations of the divergence at the ordering point in dc transport studies were only based on the assumption that an S-shaped anomaly in de current-voltage characteristics marks current-induced dynamic ordering of driven vortices. In $\mathrm{NbSe}_{2}$ crystals, ${ }^{1,2}$ the inflection anomaly is observed in the peak effect regime, where the critical current shows a peak in the vicinity of the second critical field $H_{c 2}$. Meanwhile, in amorphous $(\alpha-)$ MoGe thin films, ${ }^{18}$ instead of the peak effect, the anomaly is accompanied by a peak in the "dynamic" critical current, which characterizes a change of dynamic friction of vortices in the flux-flow state. These studies claimed the divergence of the ordering current around these peak behaviors and suggested the relevance of the thermal melting to the peaks of the static or dynamic pinning force. However, a study of scanning Hall microscopy has shown that geometrical inhomogeneity in sample edges leads to a macroscopic coexistence of ordered and disordered flow states and their dynamic evolutions with transport current may result in similar S-shaped current-voltage characteristics. ${ }^{19,20}$ Thus, it has remained controversial if the $\mathrm{S}$ anomaly marks the dynamic order of driven vortices.

Recently, mode locking (ML) has proved to be a powerful experimental technique for studying the dynamic ordering of driven vortex matter. ML is a dynamic resonance between internal lattice modes of a driven lattice and an ac drive superimposed on top of dc drive..$^{21-27}$ This technique directly probes the shear rigidity of driven vortices as a function of the velocity and allows us to find the dynamic ordering (or melting) point from the onset (or disappearance) of ML resonance. As demonstrated in a recent ML study on $\mathrm{NbSe}_{2}$ crystals, the ordering velocity exhibits a steep increase in the peak effect regime and this defines unambiguously the thermal melting point for driven vortex lattice. ${ }^{27}$

To elucidate the relevance of thermal melting to the peak behaviors of the critical current and the dynamic critical current, we will present in this paper a systematic study of ML and $\mathrm{dc}$ transport measurements on both $\alpha$-MoGe films and $\mathrm{NbSe}_{2}$ crystals over wide magnetic field and/or temperature ranges. We will show how the ordering frequency (velocity) grows with field and diverges in the peak effect regime. We will map out field-temperature phase diagrams and show how the divergent field, together with the peak fields of the critical current and the dynamic critical current, grows with lowering temperature. We will discuss influences of quenched disorder and thermal fluctuations on the onset of ML resonance and compare the divergent points to the thermodynamic melting curve of the ideal vortex lattice proposed by a quantitative Ginzburg-Landau (GL) model. ${ }^{28} \mathrm{~A}$ brief comment on the peak behavior of the dynamic critical current will be made.

\section{EXPERIMENTAL}

Amorphous $\mathrm{Mo}_{1-x} \mathrm{Ge}_{x}$ films $(x=0.22 \pm 0.01)$ were sputtered on silicon substrates mounted on a rotating copper 
TABLE I. Properties of amorphous MoGe films and $\mathrm{NbSe}_{2}$ crystals.

\begin{tabular}{lcccccccc}
\hline \hline Material & Sample & $\begin{array}{c}T_{c} \\
(\mathrm{~K})\end{array}$ & $\begin{array}{c}\rho_{n}(8 \mathrm{~K}) \\
(\mu \Omega \mathrm{m})\end{array}$ & $\begin{array}{c}-\mu_{0} d H_{c 2} /\left.d T\right|_{T_{c}} \\
(\mathrm{~T} / \mathrm{K})\end{array}$ & $\begin{array}{c}l \\
(\mathrm{~mm})\end{array}$ & $\begin{array}{c}w \\
(\mathrm{~mm})\end{array}$ & $\begin{array}{c}d \\
(\mu \mathrm{m})\end{array}$ & $G_{i}$ \\
\hline MoGe & MG 1 & 6.11 & 1.84 & 2.6 & 1.2 & 0.3 & 0.33 & $8.9 \times 10^{-5}$ \\
& MG 2 & 6.05 & 1.72 & 2.7 & 1.2 & 0.3 & 0.33 & $8.1 \times 10^{-5}$ \\
$\mathrm{NbSe}_{2}$ & NS 1 & 7.1 & 0.040 & 0.75 & 0.8 & 0.4 & $1.8^{\mathrm{a}}$ & $6.1 \times 10^{-7}$ \\
& NS 2 & 7.2 & 0.034 & 0.72 & 0.72 & 0.63 & $0.9^{\mathrm{a}}$ & $5.8 \times 10^{-7}$ \\
\hline \hline
\end{tabular}

The thickness was estimated from the normal resistance at room temperature $R(295 \mathrm{~K})$ by $d$ $=(l / w) \rho_{n}(295 \mathrm{~K}) / R(295 \mathrm{~K})$ with $\rho_{n}(295 \mathrm{~K})=1.2 \mu \Omega \mathrm{m}$ (Ref. 27).

stage held at room temperature by water cooling. The films were structured into a Hall-bar shape by a lift-off technique. After partly depositing silver on top of the films, thin gold wires were connected to the films by using small indium pieces. We used two films (MG 1 and MG 2) with nearly identical geometry $(1.2 \mathrm{~mm}$ in length $l, 0.3 \mathrm{~mm}$ in width $w$, and $0.33 \mu \mathrm{m}$ in thickness $d$ ) and material parameters of superconducting transition temperature $T_{c}$, normal resistivity $\rho_{n}$, and a slope of the second critical field near $T_{c} S\left(=\mu_{0} d H_{c 2} /\left.d T\right|_{T_{c}}\right)$, which are given in Table I. We determined $T_{c}$ from resistive transition by Aslamazov-Larkin fluctuation theory. ${ }^{29} \mathrm{H}_{c 2}$ for magnetic field perpendicular to the films was determined from the lowest-Landau-level (LLL) scaling analysis of fluctuation conductivity. ${ }^{30,31}$ The average composition for the films was obtained by the electron probe microanalyzer.

We also used pure $2 \mathrm{H}-\mathrm{NbSe}_{2}$ single crystals grown by an iodine vapor transport method. ${ }^{32}$ The thinnest platelets with optically flat surfaces on both sides were cut in a bar shape. We glued these on silicon substrates with negative resist and cleaved them without observable surface damage. After deposition of silver partly on crystal surface, thin gold wires were connected to voltage and current contacts by silver paste and indium solder, respectively. The geometry and material parameters for two cleaved crystals (NS 1 and NS 2) used in this study were also summarized in Table I. $T_{c}$ was determined by the midpoint of the resistive transition, which has a transition width of about $50 \mathrm{mK}$ between $10 \%$ and $90 \%$ of the normal-state resistance at $8 \mathrm{~K}$, just above $T_{c}$. Residual resistance ratio defined by $R(295 \mathrm{~K}) / R(8 \mathrm{~K})$ is about 30. We applied magnetic field perpendicular to the $a b$ plane of the crystals. $H_{c 2}$ was determined from the intersection between the linear extrapolation of flow resistance and the normal resistance. ${ }^{33}$ The thickness of the cleaved crystals was estimated from the room-temperature resistance by $d$ $=(l / w) \rho_{n}(295 \mathrm{~K}) / R(295 \mathrm{~K})$ using the room-temperature resistivity $\rho_{n}(295 \mathrm{~K})=1.2 \times 10^{-6} \Omega \mathrm{m}$ determined before. ${ }^{27}$

We used Oxford cryostat with a VTI inset and a 17/19 superconducting magnet. We glued a sample on a copper stage at the bottom of our measurement insert, and measured temperature by using a calibrated Cernox sensor attached to the stage. For measurements below $4.2 \mathrm{~K}$, the sample was immersed in liquid of ${ }^{4} \mathrm{He}$ introduced in the VTI insert. We regulated the vapor pressure of the liquid by using a throttle valve, a capacitance manometer, a proportional-integralderivative controller, and a rotary pump. Above $4.2 \mathrm{~K}$, we used a temperature controller (Cryocon 62), which regulates temperature of the vaporizer at the bottom of the VTI insert and that of the sample stage of the measurement insert. We employed four-terminal pair method with a precision $L C R$ meter (HP 4285A/001) and nanovoltmeters (Keithley 2182).

The sample was wired with four coaxial cables of which guards are connected. For a precise measurement of the ac current in the frequency range of $0.1-30 \mathrm{MHz}$, we calibrated the coaxial cables in two steps: First, we made open and load calibrations by unwiring and wiring a $100 \Omega$ chip resistor to the cables at the sample stage, respectively. Then, the sample was connected to the cables and cooled to the lowest temperature of $\sim 1.2 \mathrm{~K}$, which is far below $T_{c}$ of $\alpha$-MoGe films and $\mathrm{NbSe}_{2}$ crystals used in this study. Ensuring negligible contact resistance and undetectable resistance of the sample, we made short calibration as function of frequency as the second step.

During the ML measurement, we probed the dc voltage by ramping the dc current up or down with a superimposed ac current on top. The dc current value was obtained by measuring the dc voltage over a standard resistor. Other dc transport measurements of resistive transition and dc currentvoltage $(I-V)$ characteristics without ac current were performed by using a dc source (Keithley 220) and nanovoltmeters with proper filtering.

\section{RESULTS AND DISCUSSIONS}

Figure 1 displays a plot of the critical current $I_{c}$ vs field measured in $1.96 \mathrm{~K}$ for MG 1 . Here, $I_{c}$ is determined from dc $I-V$ characteristics by a voltage criterion of $1 \mu \mathrm{V}$. As observed, on increasing magnetic field, $I_{c}$ starts to increase around $5.5 \mathrm{~T}$ (the onset field) and exhibits a broad peak around $7 \mathrm{~T}\left(=\mu_{0} H_{p}\right)$. Above $7 \mathrm{~T}$, it drops rapidly and vanishes around $7.8 \mathrm{~T}$. We also plot the dynamic critical current $I_{c, d y n}$, obtained by extrapolating the flux-flow behavior linearly to the zero voltage (for definition, see the inset of Fig. 1). This characterizes the pinning strength (or dynamic friction) for driven vortices in the flux-flow state. As observed, $I_{c, d y n}$ exhibits a pronounced peak against field: It starts to show a rapid increase around $7 \mathrm{~T}$ and obtains a sharp maximum at about $7.6 \mathrm{~T}\left(=\mu_{0} H_{p, d y n}\right)$. Interestingly, the peak field (onset field) of the dynamic critical current does not coincide with the corresponding peak (onset) field for the critical current. The former appears in the higher part of the peak effect regime of the latter, i.e., $H_{p, d y n}>H_{p}$. Such peak anomaly of 


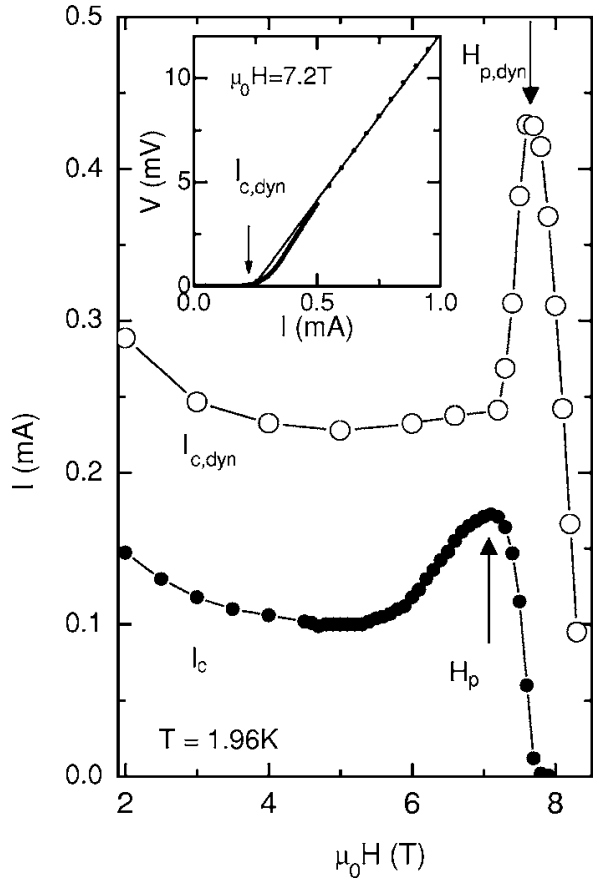

FIG. 1. Magnetic-field dependence at $1.96 \mathrm{~K}$ of critical current $I_{c}$ (solid circles) and dynamic critical current $I_{c, d y n}$ (open circles) for an $\alpha$-MoGe film (MG 1). In the inset, an $I$ - $V$ curve measured at 7.2 $\mathrm{T}$ is given. The solid line represents the linear extrapolation of the flux-flow behavior to the zero voltage. As indicated by an arrow, the intersectional point defines $I_{c, d y n}$.

the dynamic critical current is also observed in $\mathrm{NbSe}_{2}$ crystals. In the conventional picture, the peak in the critical current marks the pinning induced, structural transformation from weakly disordered to amorphous arrangement of vortices. ${ }^{36-38}$ Thus, from a phenomenological point of view, another structural transformation in the flux-flow state may occur at the peak in the dynamic critical current.

\section{A. Mode locking}

In order to explore the dynamic structural change in the flux-flow state, we have employed the ML technique. To show typical ML resonant features, we first present ML results below the peak effect regime. Shown in Fig. 2 is a series of $I-V$ curves of MG 2 measured with superimposed $10 \mathrm{MHz}$ ac current of various amplitudes at $4.2 \mathrm{~K}$ and a field of $2.0 \mathrm{~T}\left[<\mu_{0} H_{p}(4.2 \mathrm{~K})=3.0 \mathrm{~T}\right]$. A curve denoted as DC represents the pure dc $I-V$ curve without ac current $I_{a c}$. The application of $I_{a c}$ induces clear ML steps at equidistant voltages denoted by $p / q=1 / 1$ and $2 / 1$. The first step corresponds to the fundamental $(p / q=1 / 1)$, and the other is a higher harmonic $(p / q=2 / 1)$. Also even higher harmonics are observed at higher voltages, but are not shown. Because of the ML resonant condition of $v=(p / q) f a$ with integers $p, q$ and the lattice spacing of vortex lattice $a$, these ML voltages are directly proportional to the resonant frequency of ac current according to $V_{p / q}=v B l=(p / q) \Phi_{0} f l / a_{\perp}$ with the row spacing of vortex lattice $a_{\perp}\left(=\Phi_{0} / a B\right)$ and the vortex density $B .{ }^{27}$ Step width at the fundamental (and also harmonic) ML

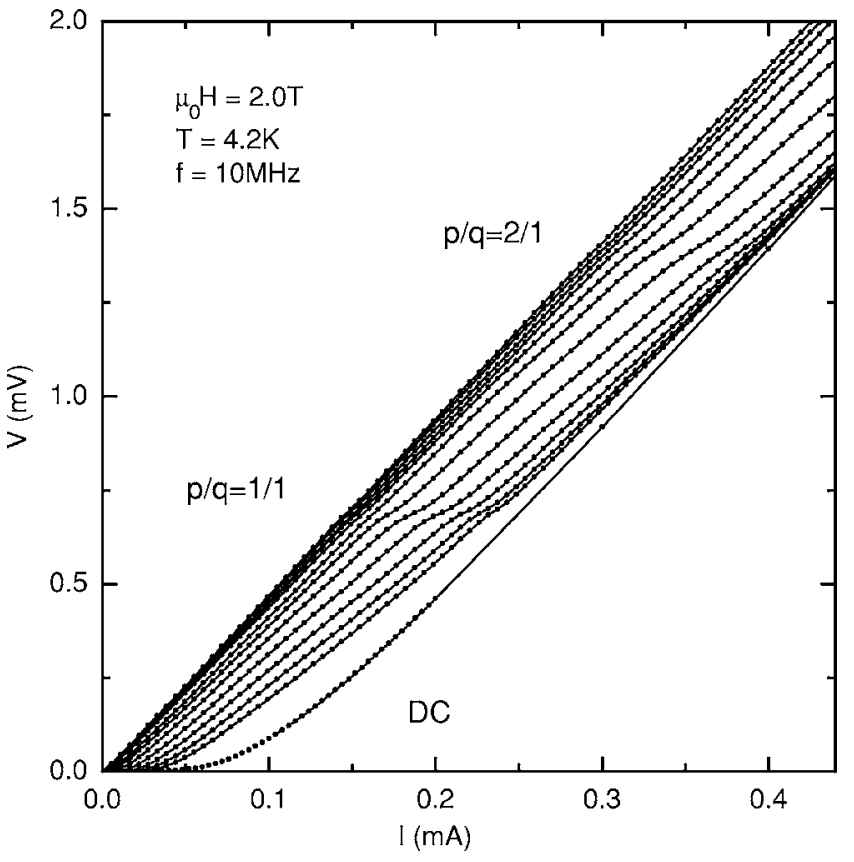

FIG. 2. A set of de $I-V$ curves for an $\alpha$-MoGe film (MG 2) measured at $2.0 \mathrm{~T}$ in $4.2 \mathrm{~K}$ with superimposed ac currents (amplitude $3.3,2.6,2.1,1.6,1.3,1.0,0.82,0.65,0.52,0.41,0.33$, and $0.26 \mathrm{~mA}$ from left to right). A curve denoted as DC indicates the pure dc $I-V$ curve. Application of ac induces clear mode-locking current steps at equidistant voltages denoted by $p / q=1 / 1$ and $1 / 2$.

resonance varies with $I_{a c}$, exhibiting an oscillatory behavior like squared Bessel function of the first kind of the first (higher) order. ${ }^{26}$ Since the oscillatory period depends on frequency, temperature, and magnetic field, we use for consistency in the following analysis the maximum width $\Delta I_{\max }$ of the fundamental resonance as the amplitude of the ML step width.

In Fig. 3(a), we show how $\Delta I_{\max }$ varies with frequency. At lower frequencies $(<10 \mathrm{MHz})$, the ML step width increases linearly with $f$, while at higher frequencies $(>10 \mathrm{MHz})$, it levels off and becomes independent of $f$. Such behavior is reasonably described by an empirical function of $\Delta I_{\max }$ $=I_{s} \tanh \left(f / 0.7 f_{p}\right)$, displayed by the solid curve. ${ }^{26}$ From the fit, we obtain a pinning frequency $f_{p}=13 \mathrm{MHz}$ and a saturated current width $I_{s}=16 \mu \mathrm{A} .{ }^{39}$ However, the ML width at lower frequencies deviates downward from the empirical function. A linear extrapolation of the data indicated by the dotted line seems to reveal a threshold behavior of the ML width, i.e., there is a finite frequency (velocity) above which the ML resonance can be observed.

Such threshold behavior is pronounced in the peak effect regime, as can be seen in Fig. 3(b), where the frequency dependence of the ML width measured at $4.2 \mathrm{~K}$ and magnetic fields of $3.25,3.4$, and $3.45 \mathrm{~T}$ is shown (above $\mu_{0} H_{p}$ $=3.0 \mathrm{~T}$ ). For instance, the linear extrapolation at $3.25 \mathrm{~T}$ clearly displays the threshold behavior at an onset frequency $f_{c} \approx 1.4 \mathrm{MHz}$ denoted by an arrow. Below $f_{c}$, no ML resonance feature is detectable at any of the ac amplitudes, indicating the absence of shear rigidity in the driven vortex matter. Thus, $f_{c}$ marks a dynamic ordering frequency (or 

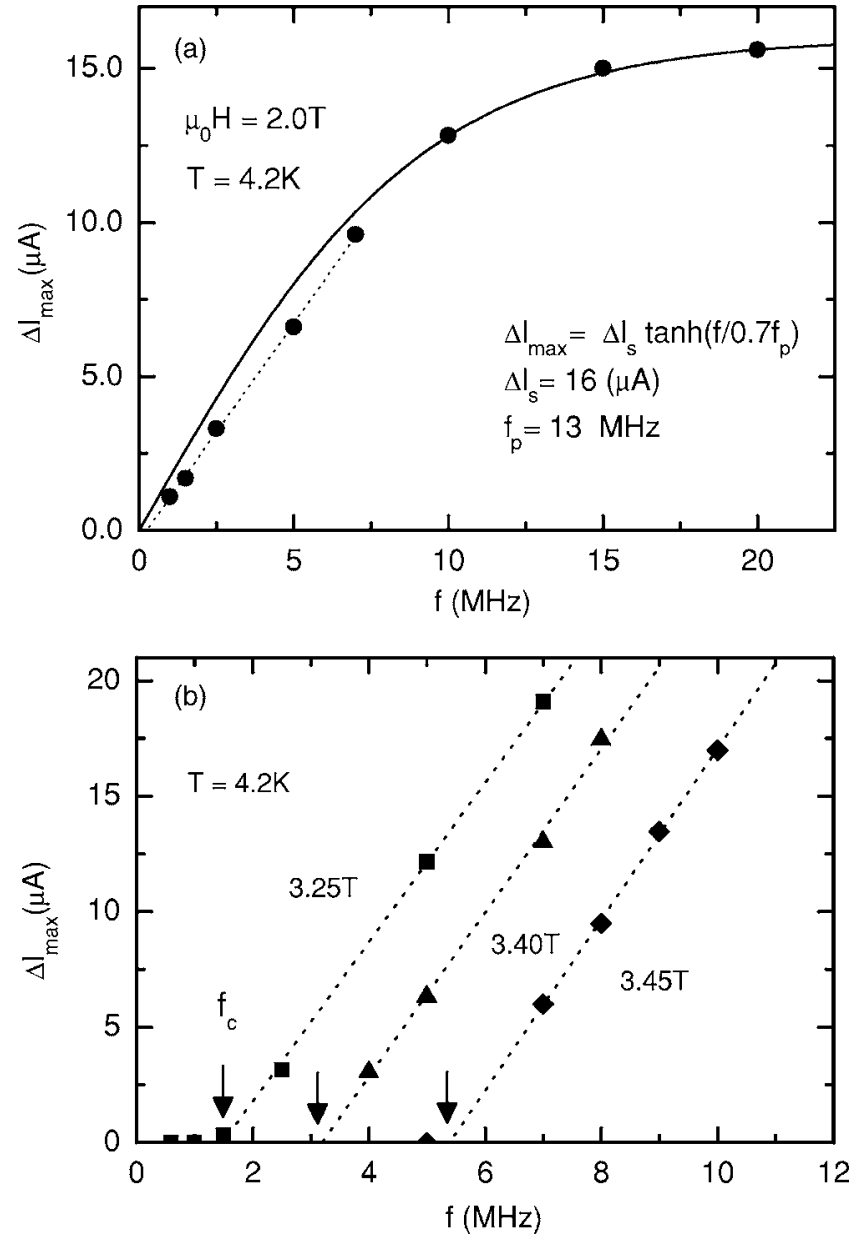

FIG. 3. Frequency dependence of maximum current width $\Delta I_{\max }$ of the fundamental ML step for an $\alpha$-MoGe film (MG 2) measured (a) at $2.0 \mathrm{~T}$, far below the peak effect regime, and (b) at fields of $3.25,3.40$, and $3.45 \mathrm{~T}$ in the peak effect regime in $4.2 \mathrm{~K}$. A solid curve represents an empirical function for the ML width given in text (Ref. 26). Dotted lines represent the linear low-frequency behavior of the ML width. Arrows mark the onset frequency $f_{c}$ for ML resonance.

velocity). ${ }^{25,26}$ When field is increased by $0.15 \mathrm{~T}$ to $3.4 \mathrm{~T}, f_{c}$ increases by $1.7 \mathrm{MHz}$ to $\approx 3.1 \mathrm{MHz}$. With further increase of field by only $0.05 \mathrm{~T}$ to $3.45 \mathrm{~T}, f_{c}$ shows a rapid increase by $\approx 2.2 \mathrm{MHz}$ and becomes $\approx 5.3 \mathrm{MHz}$. In slightly higher fields, an even more rapid increase of $f_{c}$ is observed.

We summarize results of the onset frequency at $4.2 \mathrm{~K}$ in a semilogarithmic plot of $f_{c}$ vs magnetic field, displayed in Fig. 4(b). In low fields, $f_{c}$ (depicted by solid diamonds) increases slowly with field. Around the peak field of the critical current $(=3.0 \mathrm{~T})$, it turns to a rapid increase, followed by a small knee around 3.2 T. After the knee, it exhibits a diverging behavior toward just above $3.5 \mathrm{~T}$. This is also displayed in a linear plot given in the inset of Fig. 4(b). The open symbols represent the onset frequency determined from the magnetic-field dependence of the ML width. ${ }^{27}$ Both data sets show the same diverging behavior of $f_{c}(H)$.

The divergence of $f_{c}$ is in qualitative agreement with the dynamic ordering picture proposed by Koshelev and

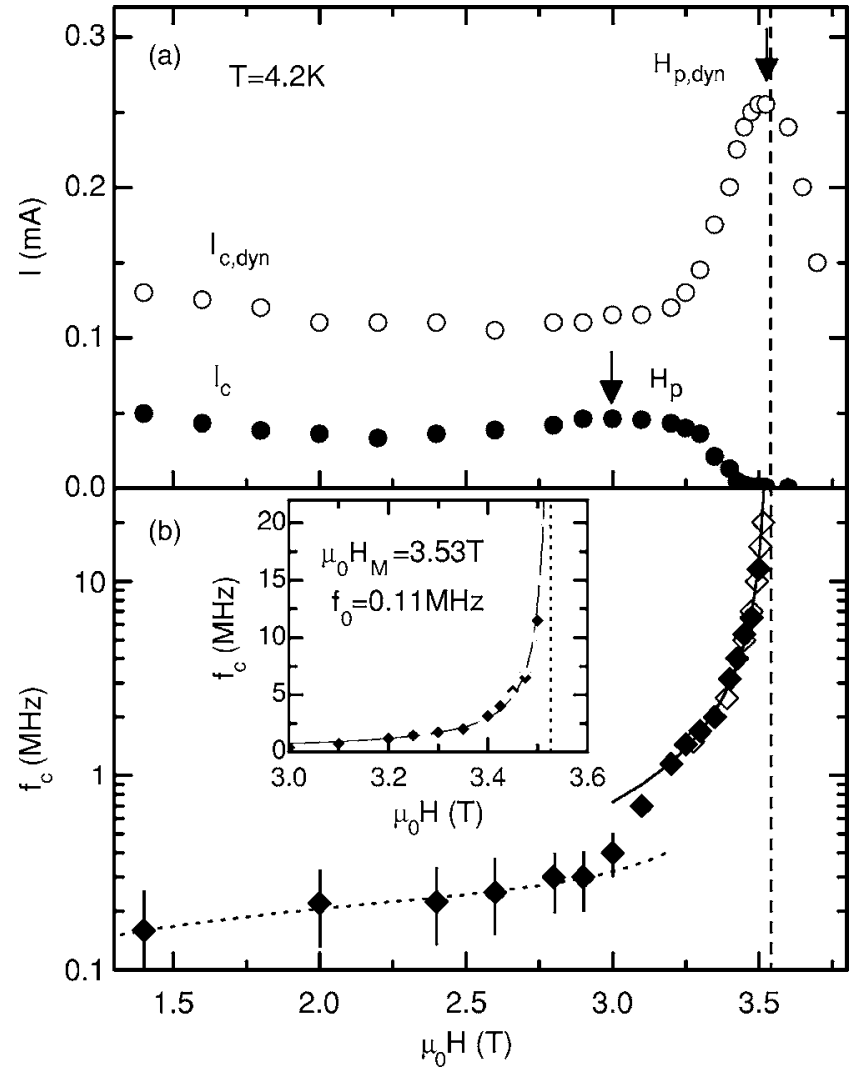

FIG. 4. Magnetic-field dependence of (a) critical current $I_{c}$ and dynamic critical current $I_{c, d y n}$ and (b) the onset frequency $f_{c}$ for an $\alpha$-MoGe film (MG 2) at 4.2 K. Peak points of the critical current and the dynamic critical current are indicated by arrows in (a). The inset of (b) displays the plot of $f_{c}$ on a linear frequency scale.

Vinokur. ${ }^{3}$ The dynamic crystallization frequency can be given by ${ }^{25,26}$

$$
f_{c}=\frac{v_{c}}{a}=\sqrt{\frac{3}{2 \pi}} \frac{\gamma_{u} \rho_{f}}{\Phi_{0}^{2} a^{2} d k_{B} T_{M}} \frac{1}{\left(1-T / T_{M}\right)},
$$

with a disorder parameter $\gamma_{u}$ and the flux-flow resistivity $\rho_{f}$. This expression qualitatively explains the divergence of $f_{c}$ (or $v_{c}$ ) against temperature observed in an $\alpha$-MoGe film ${ }^{40}$ and also in mesoscopic flow channels. ${ }^{25}$ Moreover, as displayed by the solid curve in Fig. 4(b) (also in the inset), we find that the diverging behavior of $f_{c}$ against magnetic field is approximated well by a similar diverging function of $f_{c}$ $=f_{0} /\left(1-H / H_{M}\right)$ with $\mu_{0} H_{M}=3.53 \mathrm{~T}$ (indicated by the broken vertical line) and $f_{0}=0.11 \mathrm{MHz}$ over more than one decade in frequency range. These qualitative agreements allow us to identify the field (or temperature) of the divergence as the thermal melting point of the coherently flowing vortex lattice. ${ }^{27}$ Away from $H_{M}$ at lower fields, the situation is different. Here, the onset of ML resonance occurs at small frequencies (velocities) and the influence of quenched disorder dominates the ordering transition. The contribution of quenched disorder on $f_{c}$ may be determined from the following argument: The disorder parameter is given by the amplitude of the pinning correlation function of the random pinning potential which decays over the pinning radius $r_{p}{ }^{3}$ 
Using the maximum pinning force $F_{p}$ per pinning site, we may write

$$
\gamma_{u}=\left[N_{p} F_{p}^{2}(a / 2 \pi)^{2} / 2\right]\left(r_{p}\right)^{2} \approx W a^{6} d / 16 \pi^{2},
$$

where $N_{p}\left(=a^{2} d n_{p}\right)$ is the number of pinning sites per vortex line volume, $n_{p}$ is the density of pinning centers, $W$ $\left(=n_{p} F_{p}^{2} / 2\right)$ is the Larkin-Ovchinnikov pinning parameter, ${ }^{41}$ and $r_{p} \approx a / 2 .{ }^{42}$ Assuming $T_{M}-T \approx \mu_{0}\left(H_{M}-H\right) / S$, we find

$$
f_{c} \approx f_{0} \frac{1}{\left(1-H / H_{M}\right)},
$$

with

$$
f_{0}=\frac{1}{4 \pi^{2} \sqrt{6 \pi}} \frac{S \rho_{f}(H) W(H)}{\mu_{0} H_{M} k_{B} B^{2}} .
$$

We know that the pinning properties for $\alpha$-MoGe films are well described by the delta- $T_{c}$ pinning, i.e., $W=C b(1-b)^{2}$ with a reduced field $b\left[\equiv H / H_{c 2}(T)\right]^{42,43,38}$ The flux-flow resistivity for the amorphous films in the GL regime can be written as $\left(\rho_{f} / \rho_{n}\right)^{-1}=\widetilde{F}(b) / \sqrt{1-t}+1$ with a reduced temperature $t \equiv T / T_{c}{ }^{34}$ In fields measured here, i.e., $b=\mu_{0} H / \mu_{0} H_{c 2}$ $\geqslant 1.4 \mathrm{~T} / 4.54 \mathrm{~T}$, the function $\widetilde{F}(b)$ can be approximated by an interpolation formula $\tilde{F}(b)=(1-b)^{3 / 2}[0.43+0.69(1$ $-b)] / b{ }^{44}$ Substituting this formula into Eq. (4), together with $\mu_{0} H=B$, we find that $f_{c}$ varies with magnetic field as

$$
f_{c} \propto \frac{(1-b)^{2}}{b[1+\widetilde{F}(b) / \sqrt{1-t}]} \frac{1}{1-H / H_{M}} .
$$

As displayed by the dotted curve, this shows a slow increase with field away from $H \approx H_{M}$ and explains our results below $H_{p}$. The proportionality constant extracted from the fit provides a rough estimate for the coefficient $C$ in the expression for the pinning strength $W$, namely, $C \approx 1.2 \times 10^{-8} \mathrm{~N}^{2} / \mathrm{m}^{3}$ at $T \approx 0.7 T_{c}$. This value is reasonably consistent with values obtained for previous transport measurements on similar amorphous superconducting films. ${ }^{42,43,38}$ Just above $H_{p}, f_{c}$ switches to a rapid increase up to the knee. This behavior follows neither the diverging behavior close to $H_{M}$ displayed by a solid curve nor the low-field behavior by a dotted curve. It indicates a rapid enhancement of the disorder contribution (probably the pinning parameter $W$ ). Since the ordering frequency (velocity) around $H_{p}$ is small and the disorder effect does not diminish significantly, this jump behavior could be related to the underlying mechanism for the peak effect, i.e., the enhancement of the pinning force caused by structural disordering transformation of static vortices at $H_{p}{ }^{36-38}$

We have systematically made ML and dc transport measurements in different temperatures on both $\alpha$-MoGe films and $\mathrm{NbSe}_{2}$ crystals. Results commonly show that $f_{c}$ exhibits the jump and subsequent knee behavior around $H_{p}$. The diverging behavior of $f_{c}$ occurs always in the higher part of the peak effect regime and the divergent point lies in between the peak field and the second critical field, i.e., $H_{p}<H_{M}$ $<H_{c 2}$. Thus, the observed behavior of $f_{c}$ allows us to separate the thermal melting marked by the divergent point from the disorder induced transformation marked by $H_{p}$.
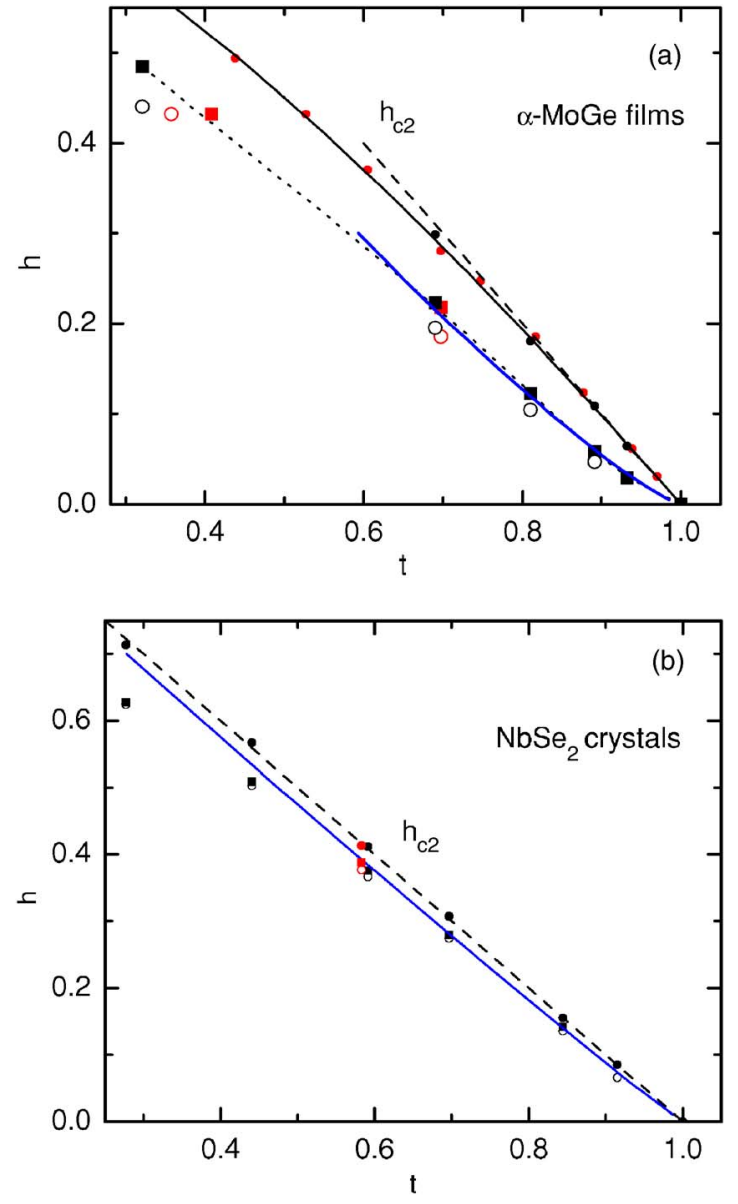

FIG. 5. (Color online) Magnetic field-temperature phase diagrams for (a) $\alpha$-MoGe films and (b) $\mathrm{NbSe}_{2}$ crystals. The magnetic field and temperature are normalized by superconducting transition temperature $T_{c}$ and the Ginzburg-Landau second critical field $H_{c 2}^{G L}(0)\left(\equiv S T_{c}\right)$. Plotted are reduced second critical fields $h_{c 2}$ (small solid circles), divergence fields $h_{M}$ of the ordering frequency (solid squares), and peak fields $h_{p}$ of the critical current (open circles). In (a), black and red colored symbols correspond to results for MG 1 and MG 2, respectively. In (b), black (red) colored symbols are for NS 1 (NS 2). In (a), a solid black curve represents the mean-field curve for the dirty limit in WHH theory (Ref. 45). For comparison, the linear dependence of $h_{c 2}$ on $1-t$ is displayed by a broken line in (a) [also in (b)]. A dotted curve in (a) is a guide for the eyes. Blue curves in (a) and (b) represent thermodynamic melting curves obtained from a quantitative GL model (Ref. 28) for MG 1 and NS 1 crystals, respectively.

\section{B. Phase diagrams}

Based on these findings, let us map out a fieldtemperature phase diagram for $\alpha$-MoGe films of MG 1 and MG 2. To show results in two films together in Fig. 5(a), magnetic field and temperature are normalized by the GL second critical field $H_{c 2}^{G L}(0)\left(\equiv S T_{c}\right)$ and $T_{c}$, respectively. For clarity, results of MG 1 and MG 2 are depicted by black and red colored symbols, respectively. Good agreement is visible in temperature dependencies of $h_{c 2}\left[\equiv H_{c 2} / H_{c 2}^{G L}(0)\right]$ plotted for the films with solid red and black circles: On lowering temperature from $t=1, h_{c 2}(t)$ increases linearly with $1-t$ dis- 
played by a broken line. For $t \leqslant 0.8, h_{c 2}(t)$ deviates downward from the linear dependence. Such behavior is well described by the mean-field curve (displayed by the solid curve) for the dirty limit in Werthamer-Helfand-Hohenberg (WHH) theory ${ }^{45}$ which is given by

$$
\operatorname{In}(t)=\Psi(1 / 2)-\Psi\left[1 / 2+\left(2 / \pi^{2}\right) h_{c 2}(t) / t\right],
$$

where $\Psi$ is the digamma function.

In Fig. 5(a), the results of $h_{M}(t)\left[\equiv H_{M} / H_{c 2}^{G L}(0)\right]$ for the films are also depicted by solid squares. Focusing on results of MG 1, we find that on lowering temperature from $t=1$, $h_{M}(t)$ increases slowly and the field distance between $h_{M}(t)$ and $h_{c 2}(t)$ becomes larger. With further lowering $t, h_{M}(t)$ gradually increases faster and it becomes nearly parallel to $h_{c 2}(t)$ for $t<0.8$. Thus, the field distance between $h_{M}(t)$ and $h_{c 2}(t)$ is nearly constant for $t<0.8$. Results of MG 2 follow the $h_{M}(t)$ curve for MG 1 well. Because of the thermal melting of the flowing vortex lattice marked by $h_{M}(t)$, the regime in the phase diagram between $h_{M}(t)$ and $h_{c 2}(t)$ should represent a flowing liquid of driven vortices.

We also map out the field-temperature phase diagram for $\mathrm{NbSe}_{2}$ crystals of NS 1 and NS 2 in Fig. 5(b). $h_{c 2}(t)$ increases linearly with $1-t$ down to the lowest temperature measured. This is consistent with thermodynamic studies on $\mathrm{NbSe}_{2}$ crystals. ${ }^{46,47}$ In contrast to the results on $\alpha$-MoGe films, $h_{M}(t)$ lies just below the mean-field line except for low temperatures $(t \lesssim 0.4)$. For instance, the field distance between $h_{M}(t)$ and $h_{c 2}(t)$ at $t \sim 0.7$ is 0.02 for $\mathrm{NbSe}_{2}$ crystals, which is smaller than 0.08 for $\alpha$-MoGe films. In other words, the vortex liquid flow regime for $\mathrm{NbSe}_{2}$ crystals is much narrower than that for $\alpha$-MoGe films.

This difference should be related to the strength of thermal fluctuations, which may be characterized by the Ginzburg number given by

$$
G i=\frac{1}{2}\left(\frac{k_{B} T_{c} \gamma}{4 \pi \mu_{0} H_{c}(0)^{2} \xi_{a b}^{3}(0)}\right)^{2},
$$

where $\gamma$ is an anisotropy parameter, $H_{c}(0)$ is the thermodynamic critical field at $T=0$, and $\xi_{a b}(0)$ is the coherence length for the $a b$ plane at $T=0 .{ }^{48}$ For estimation of $G i$ on $\alpha$-MoGe films, we use the dirty-limit expression for $\mu_{0} H_{c}(0)=S T_{c} / 2.54 \kappa$ with $\kappa=3.54 \times 10^{4}\left(\rho_{n} S\right)^{1 / 2}$ (Ref. 42) and $\gamma=1$. For $\mathrm{NbSe}_{2}$ crystals, we use values of the GL thermodynamic field $\mu_{0} H_{c}(0)=0.23 \mathrm{~T}$ and $\gamma=3$, reported in the literature. ${ }^{46,47}$ As listed in Table I, $\alpha$-MoGe films have a nearly identical value of $G i \approx 8 \times 10^{-5}$, which is 2 orders of magnitude larger than that for $\mathrm{NbSe}_{2}$ crystals. This is qualitatively consistent with the broader regime of the vortex liquid flow for $\alpha$-MoGe films.

Quantitative estimation of the distance between the thermodynamic melting curve and the mean-field line can be made by using the recent quantitative GL result proposed by $\mathrm{Li}$ and Rosenstein (LR) ${ }^{28}$ In their theory, the thermodynamic melting transition sets in when the LLL-scaled temperature given by

$$
a_{T}=-a_{h}\left(\sqrt{\frac{N_{G i}}{2}} \pi t h\right)^{-2 / 3}
$$

is equal to -9.5 . Here, $N_{G i}\left(=G i / \pi^{2}\right)$ is their Ginzburg number and $a_{h}(=1-t-h)$ is the distance from the mean-field line. Substituting $G i$ for $\alpha$-MoGe films and $\mathrm{NbSe}_{2}$ crystals from Table I, we calculated how far the melting curve is located from the mean-field line $\left[1-t-h_{c 2}(t)=0\right]$. A solid blue curve in Fig. 5(a) represents the calculated melting curve for MG 1. Good agreement with $h_{M}(t)$ data is visible at high temperatures $(t \geqslant 0.6)$. We note that the melting curve at low temperatures $(t<0.6)$ is not shown since clear deviations of $h_{c 2}$ from the linear dependence, which is presumed by the model, appear at low temperatures. We also display the calculated melting curve for NS 1 in Fig. 5(b). Because of small $G i$, the calculated melting curve lies just below the $h_{c 2}(t)$ line. Again, good agreement of $h_{M}(t)$ with the melting curve is visible at high temperatures $(t \geqslant 0.6)$. Taking into account that this model is valid in temperatures not far from $t=1$, the agreements of $h_{M}(t)$ with the LR model for both MG 1 and NS 1 having more than 2 orders of magnitude difference in $G i$ are quite remarkable, providing a further link between the thermal melting point of the flowing lattice and the thermodynamic one of the static and ideal vortex lattice. We note that the influence of applied currents upon the thermodynamic properties is negligibly small. ${ }^{49}$

We also plot the peak fields $h_{p}\left[\equiv H_{p} / H_{c 2}^{G L}(0)\right]$ of the critical current (open circles) in Figs. 5(a) and 5(b). In both cases, they lie below the thermodynamic melting curve [or $\left.h_{M}(t)\right]$. This indicates that for static vortices the pinning induced, structural transformation into amorphous array of vortices occurs prior to the thermodynamic melting and therefore a crossover (or a continuous transition) from glassy to liquid states may occur around the thermodynamic melting point. We emphasize that in the higher part of the peak effect regime, the dynamic melting features discussed above are observable only when vortices are driven fast enough and that the elasticity is recovered by the dynamical ordering from the amorphous static state.

Finally, we comment on the peak behavior in the dynamic critical current. We find that all the peak points of the dynamic critical current observed for both $\alpha$-MoGe films and $\mathrm{NbSe}_{2}$ crystals coincide nearly with corresponding divergent points of $h_{M}(t)$ (not shown for clarity). This agreement is exemplified in Figs. 4(a) and 4(b), where the peak point of the dynamic critical current occurs very close to the divergence of $f_{c}$. These coincidences suggest that the peak point of the dynamic critical current could be a good indication for the thermal melting of the coherent lattice flow. However, it is not clear to us why the lower part of the peak anomaly observed in both $\alpha$-MoGe films and $\mathrm{NbSe}_{2}$ crystals changes gradually and does not show a jump at the thermal (or thermodynamic) melting point. This gradual change is possibly related to an extrinsic origin such as inhomogeneity in samples and/or geometrical influences of edge ${ }^{51}$ and surface roughness. ${ }^{52}$

\section{SUMMARY}

In summary, employing the mode-locking technique, we have presented the dynamic ordering of driven vortex matter 
in the peak effect regime of both amorphous MoGe films and $\mathrm{NbSe}_{2}$ crystals. The dynamic ordering frequency (velocity) marked by the onset of the ML resonance exhibits a sharp increase and subsequent knee behavior around the peak field of the critical current, followed by a striking diverging behavior in the higher part of the peak effect regime. The former jump behavior indicates the rapid enhancement of pinning contribution, probably originating from the underlying structural transformation of the static vortex lattice due to quenched disorder at $H_{p}$. Meanwhile, the latter diverging behavior is in good agreement with the dynamic ordering model and the divergence point is identified as the thermal melting point of the driven vortex lattice.

The thermal melting point measured at each temperature lies in between the peak field and the second critical field, and it nearly coincides with the peak point of the dynamic critical current. Mapping out the field-temperature phase diagrams for amorphous MoGe films and $\mathrm{NbSe}_{2}$ crystals, we find that the thermal melting points follow well the thermo- dynamic melting curve for the static and ideal vortex lattice over wide field and/or temperature ranges. These findings clearly separate the peak effect from thermal melting and provide a link between static and dynamic melting phenomena.

\section{ACKNOWLEDGMENTS}

N.K. thanks E. Zeldov for useful comments on electronic contacts for $\mathrm{NbSe}_{2}$ crystals. N.K. also thanks S. Bhattacharya, B. Rosenstein, G. Bel, and V. M. Vinokur for useful comments. N. K. used facilities in cryogenic center in the University of Tsukuba. This work was partly supported by the Grant in Aid for Scientific Research from MEXT (the Ministry of Education, Culture, Sports, Science and Technology) and by the 21st Century COE (Center of Excellence) Program "Promotion of Creative Interdisciplinary Materials Science for Novel Functions" under MEXT, Japan.
* Present address: Center for Research and Advancement in Higher Education, Kyushu University, 4-2-1 Ropponmatsu, Chuoh-ku, Fukuoka, Fukuoka 810-0044, Japan. FAX: +81-92-726-4792, Email address: kokubo@rc.kyushu-u.ac.jp

${ }^{1}$ S. Bhattacharya and M. J. Higgins, Phys. Rev. Lett. 70, 2617 (1993).

${ }^{2}$ M. J. Higgins and S. Bhattacharya, Physica C 257, 232 (1996).

${ }^{3}$ A. E. Koshelev and V. M. Vinokur, Phys. Rev. Lett. 73, 3580 (1994).

${ }^{4}$ P. Le Doussal and T. Giamarchi, Phys. Rev. B 57, 11356 (1998).

${ }^{5}$ L. Balents, M. C. Marchetti, and L. Radzihovsky, Phys. Rev. B 57, 7705 (1998).

${ }^{6}$ S. Scheidl and V. M. Vinokur, Phys. Rev. E 57, 2574 (1998).

${ }^{7}$ S. Scheidl and V. M. Vinokur, Phys. Rev. B 57, 13800 (1998).

${ }^{8}$ M. C. Faleski, M. C. Marchetti, and A. A. Middleton, Phys. Rev. B 54, 12427 (1996).

${ }^{9}$ A. B. Kolton, D. Dominguez, and N. Gronbech-Jensen, Phys. Rev. Lett. 83, 3061 (1999).

${ }^{10}$ C. J. Olson, C. Reichhardt, and F. Nori, Phys. Rev. Lett. 81, 3757 (1998).

${ }^{11}$ H. Fangohr, S. J. Cox, and P. A. J. de Groot, Phys. Rev. B 64, 064505 (2001)

${ }^{12}$ M. Chandran, R. T. Scalettar, and G. T. Zimanyi, Phys. Rev. B 67, 052507 (2003).

${ }^{13}$ V. Gotcheva, Y. Wang, A. T. J. Wang, and S. Teitel, Phys. Rev. B 72, 064505 (2005).

${ }^{14}$ J. Shelten, G. Ullmaier, and H. Lippman, Phys. Rev. B 12, 1772 (1975).

${ }^{15}$ U. Yaron, P. L. Gammel, D. A. Huse, R. N. Kleiman, C. S. Oglesby, E. Bucher, B. Batlogg, D. J. Bishop, K. Mortensen, and K. N. Clausen, Nature (London) 376, 753 (1995).

${ }^{16}$ M. Marchevsky, J. Aarts, P. H. Kes, and M. V. Indenbom, Phys. Rev. Lett. 78, 531 (1997).

${ }^{17}$ F. Pardo, F. de la Cruz, P. L. Gammel, E. Bucher, and D. J. Bishop, Nature (London) 399, 665 (1998).

${ }^{18}$ M. C. Hellerqvist, D. Ephron, W. R. White, M. R. Beasley, and A.
Kapitulnik, Phys. Rev. Lett. 76, 4022 (1996).

${ }^{19}$ M. Marchevsky, M. J. Higgins, and S. Bhattacharya, Nature (London) 409, 591 (2001).

${ }^{20}$ Y. Paltiel, Y. Myasoedov, E. Zeldov, G. Jung, M. L. Rappaport, D. E. Feldman, M. J. Higgins, and S. Bhattacharya, Phys. Rev. B 66, 060503(R) (2002).

${ }^{21}$ A. T. Fiory, Phys. Rev. Lett. 27, 501 (1971).

${ }^{22}$ P. Martinoli O. Daldini, C. Leemann, and E. Stocker, Solid State Commun. 17, 207 (1975).

${ }^{23}$ L. Van Look, E. Rosseel, M. J. Van Bael, K. Temst, V. V. Moshchalkov, and Y. Bruynseraede, Phys. Rev. B 60, R6998 (1999).

${ }^{24}$ N. Kokubo, R. Besseling, V. M. Vinokur, and P. H. Kes, Phys. Rev. Lett. 88, 247004 (2002).

${ }^{25}$ R. Besseling, N. Kokubo, and P. H. Kes, Phys. Rev. Lett. 91, 177002 (2003).

${ }^{26}$ N. Kokubo, R. Besseling, and P. H. Kes, Phys. Rev. B 69, 064504 (2004).

${ }^{27}$ N. Kokubo, K. Kadowaki, and K. Takita, Phys. Rev. Lett. 95, 177005 (2005).

${ }^{28}$ D. Li and B. Rosenstein, Phys. Rev. B 65, 220504(R) (2002).

${ }^{29}$ L. G. Aslamazov and A. I. Larkin, Phys. Lett. 26A, 238 (1968).

${ }^{30}$ M. H. Theunissen and P. H. Kes, Phys. Rev. B 55, 15183 (1997).

${ }^{31}$ N. Kokubo, J. Aarts, and P. H. Kes, Phys. Rev. B 64, 014507 (2001).

${ }^{32}$ K. Takita and K. Masuda, J. Low Temp. Phys. 58, 127 (1985).

${ }^{33}$ In our $\mathrm{NbSe}_{2}$ crystals, the resistive transition measured at small current exhibits a pronounced broadening due to possible influence of the surface superconductivity along lateral surfaces of the crystals (Ref. 35). This cannot be explained by the superconducting fluctuations because the critical fluctuation region for the $\mathrm{NbSe}_{2}$ crystals is very narrow. Thus, we took different approach for determining $H_{c 2}$ as done in Refs. 34 and 35.

${ }^{34}$ P. Berghuis and P. H. Kes, Phys. Rev. B 47, 262 (1993).

${ }^{35}$ G. D'Anna, P. L. Gammel, A. P. Ramirez, U. Yaron, C. S. Oglesby, E. Bucher, and D. J. Bishop, Phys. Rev. B 54, 6583 (1996). 
${ }^{36}$ A. M. Troyanovski, M. van Hecke, N. Saha, J. Aarts, and P. H. Kes, Phys. Rev. Lett. 89, 147006 (2002).

${ }^{37}$ Y. Paltiel, E. Zeldov, Y. Myasoedov, M. L. Rappaport, G. Jung, S. Bhattacharya, M. J. Higgins, Z. L. Xiao, E. Y. Andrei, P. L. Gammel, and D. J. Bishop, Phys. Rev. Lett. 85, 3712 (2000).

${ }^{38}$ R. Wördenweber, P. H. Kes, and C. C. Tsuei, Phys. Rev. B 33, 3172 (1986).

${ }^{39}$ The pinning frequency is much larger than $f_{p}=J_{c} \rho_{f} / a B$ $\approx 3 \mathrm{MHz}(8.2 \mathrm{MHz})$ estimated from the (dynamic) critical current, flux-flow resistivity $\rho_{f}$, and $B=\mu_{0} H$. This difference would be explained by possible distribution in the pinning frequency due to plasticity in driven vortices.

${ }^{40}$ N. Kokubo, S. Okayasu, and K. Kadowaki, AIP Conf. Proc. 850, 853 (2006).

${ }^{41}$ A. I. Larkin and Yu. Ovchinnikov, J. Low Temp. Phys. 34, 409 (1979).

${ }^{42}$ P. H. Kes and C. C. Tsuei, Phys. Rev. B 28, 5126 (1983).

${ }^{43}$ R. Wördenweber, A. Prumboom, and P. H. Kes, J. Low Temp. Phys. 70, 253 (1988).

${ }^{44}$ A. I. Larkin and Yu. Ovchinnikov, in Nonequilibrium Superconductivity, edited by P. L. Langenberg and A. I. Larkin (NorthHolland, Amsterdam, 1986).

${ }^{45}$ N. Werthamer, E. Helfand, and P. C. Hohenberg, Phys. Rev. 147,
295 (1966).

${ }^{46}$ N. Toyota, H. Nakatsuji, K. Noto, A. Hoshi, N. Kobayashi, Y. Muto, and Y. Onodera, J. Low Temp. Phys. 25, 485 (1976).

${ }^{47}$ N. Kobayashi, K. Noto, and Y. Muto, J. Low Temp. Phys. 27, 217 (1977).

${ }^{48}$ G. Blatter, M. V. Feigel'man, V. B. Geshkenbein, A. I. Larkin, and V. M. Vinokur, Rev. Mod. Phys. 66, 1125 (1994).

${ }^{49}$ The influence of the applied currents upon thermodynamic properties for the isotropic superconductor may be characterized by the dimensionless velocity $\widetilde{v}=6 \pi \mu_{0} V \xi^{3} \kappa^{2} / l \Phi_{0} \rho_{n} h(1-t)$ (Ref. 50). Substituting material parameters and the ML voltage for $10 \mathrm{MHz}$ by the thermal melting point, we find $\widetilde{v} \approx 4 \times 10^{-5}$ at $h_{M}(0.7) \approx 0.22$ for MG 2 and $\widetilde{v} \approx 3 \times 10^{-4}$ at $h_{M}(0.7) \approx 0.28$ for NS 1 . These are negligibly small compared to the distance from the mean-field line $a_{h}(t=0.7) \approx 0.08$ and 0.02 for MG 2 and NS 1 , respectively.

${ }^{50} \mathrm{G}$. Bel and B. Rosenstein, arXiv:cond-mat/0509677 (unpublished).

${ }^{51}$ Z. L. Xiao, E. Y. Andrei, Y. Paltiel, E. Zeldov, P. Shuk, and M. Greenblatt, Phys. Rev. B 65, 094511 (2002).

${ }^{52}$ A. Pautrat, C. Goupil, Ch. Simon, D. Charalambous, E. M. Forgan, G. Lazard, P. Mathieu, and A. Brûlet, Phys. Rev. Lett. 90, 087002 (2003). 